\title{
System Design of the Remote Controlled Intelligent Flowerpot
}

\author{
Li Feng $^{1, \mathrm{a}}$, Ning Tingzhou ${ }^{2, \mathrm{~b}}$, Shi Long ${ }^{1, \mathrm{c}}$ and Pang Guoguang ${ }^{1, \mathrm{~d}}$ \\ ${ }^{1}$ School of Mechanical Engineering, Shandong Huayu University of Technology, Dezhou 253034, China \\ ${ }^{2}$ College of Engineering, China Agriculture University, Beijing 100083, China \\ awfjh@163.com, ’ning-tingzhou@163.com, 'hysl132237@163.com, ${ }^{\mathrm{a}} 18765472013 @ 163 . c o m$
}

Keywords: remote control, intelligent flowerpot, GSM-SM modem.

\begin{abstract}
In order to meet the needs of intelligent products, the system of remote controlled intelligent flowerpot is designed. For the sake of no person caring of flowerpot long time, the flowerpot system is controlled by a GSM-SM modem. Experiments show that this remote controlled intelligent flowerpot system is not only suitable for the planting of small potted plants, but also can be used in the fields of growth of field crop, greenhouse cultivation and so on.
\end{abstract}

\section{Introduction}

With the improvement of people's living standard, consumers pay more and more attention to the new intelligent products[1-4]. The quickening pace of life also urges consumers, students and tourists, but everyone does not lose the temperament of planting flowers and growing grass. Due to the reasons for going out, vacations and business trips, the flowers and plants are unattended for leaving at home or school. Therefore, to meet people's need for intelligent products, the remote controlled intelligent flowerpot was designed which is controlled by a GSM-SM modem. In addition, the automatic alarm and the response of timely growth parameter make the planting of flowers and plants carefree. The remote controlled intelligent flowerpot system is not only suitable for the planting of small potted plants, but also can be used in the fields of growth of field crop, greenhouse cultivation and so on. It is suitable for students, office workers, old people, farmers and scientific research workers, etc.

\section{Structure and Working Principle}

\subsection{Structure}

For the remote controlled intelligent flowerpot, the core control circuit is STM32F10C8T6 microcontroller. The control circuit consists of a temperature sensor and a humidity sensor, a GSMSM modem, a highlighted LED filling light circuit, and a photosensitive resistance control circuit. The whole flowerpot system is made up of additional parts, such as telescopic brackets, water pump and so on. The structure of this flowerpot is shown in Figure 1.

\subsection{Working Principle}

This remote controlled intelligent flowerpot measures the parameters of plant growth by the circuits of temperature sensor, humidity sensor and photosensitive resistance[5,6], such as temperature, humidity, light intensity. Firstly, the control system automatically alarms when parameters of plant growth are not predetermined. Secondly, this control circuits belongs to the two sensors and the photosensitive resistance start to control water pumps and let LED light work, such as automatically pumping water, automatically filling light. The unique GSM-SM modem plays a role when the person goes out. The flowerpot can be controlled remotely via short message service, which can remotely inquire about parameters of plant growth, remotely and timely reminding, remotely watering the plant and filling the light. 


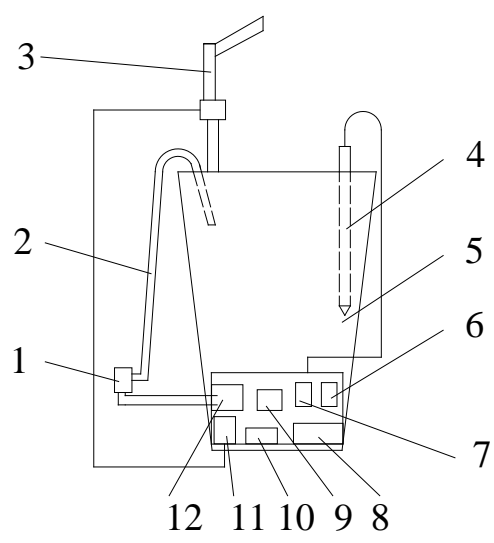

1. water pump, 2. water pipe, 3. retractable LED filling light, 4. temperature and humidity sensors, 5. flowerpot, 6. circuit of LED filling light , 7. circuit of temperature and humidity sensors, 8. micro-controller, 9. circuit of photosensitive resistance, 10. module of GSM-SM modem, 11. power switch, 12. circuit of pump control

Figure 1 Diagram of flowerpot structure

\section{Key Parts of Flowerpot and its Control Circuits}

Key parts of flowerpot includes GSM-SM modem, highlighted LCD screen, voltage stabilizer, humidity sensor, temperature sensor, STM32F10C8T6 micro-controller, water pump, which are shown in Figure 2. Control circuits of the key parts of flowerpot are given in Figure 3. The prototype of the remote controlled intelligent flowerpot is shown in Figure 4.

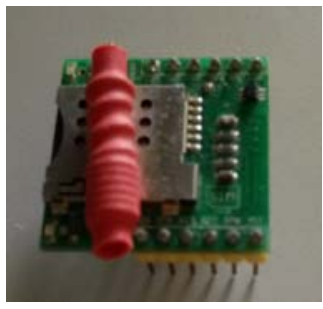

(a) GSM-SM modem

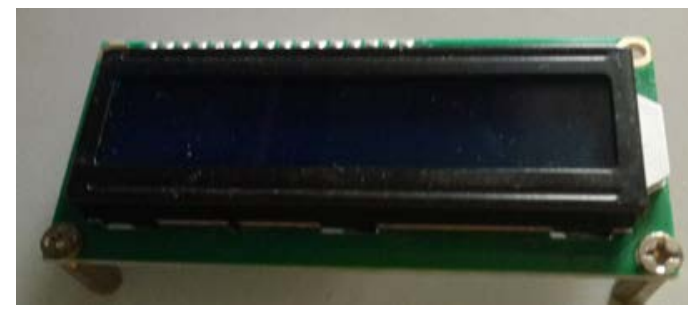

(b) Highlighted LCD screen

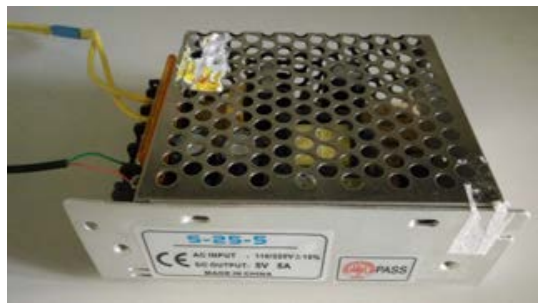

(c) Voltage stabilizer

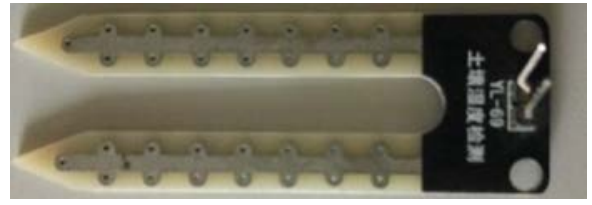

(d) humidity sensor

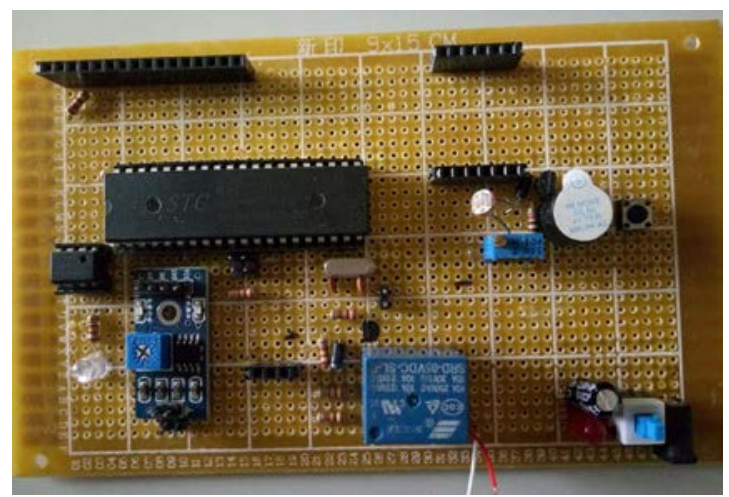

(f) STM32F10C8T6 micro-controller

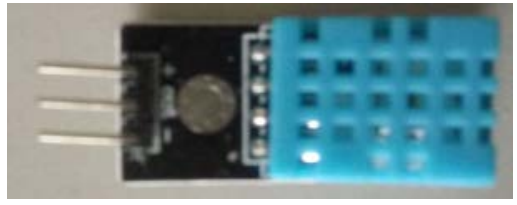

(e) temperature sensor

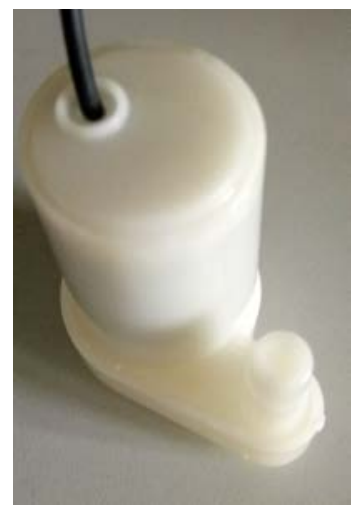

(g) $1.5 \mathrm{~V}$ water pump

Figure 2 Key parts of flowerpot 


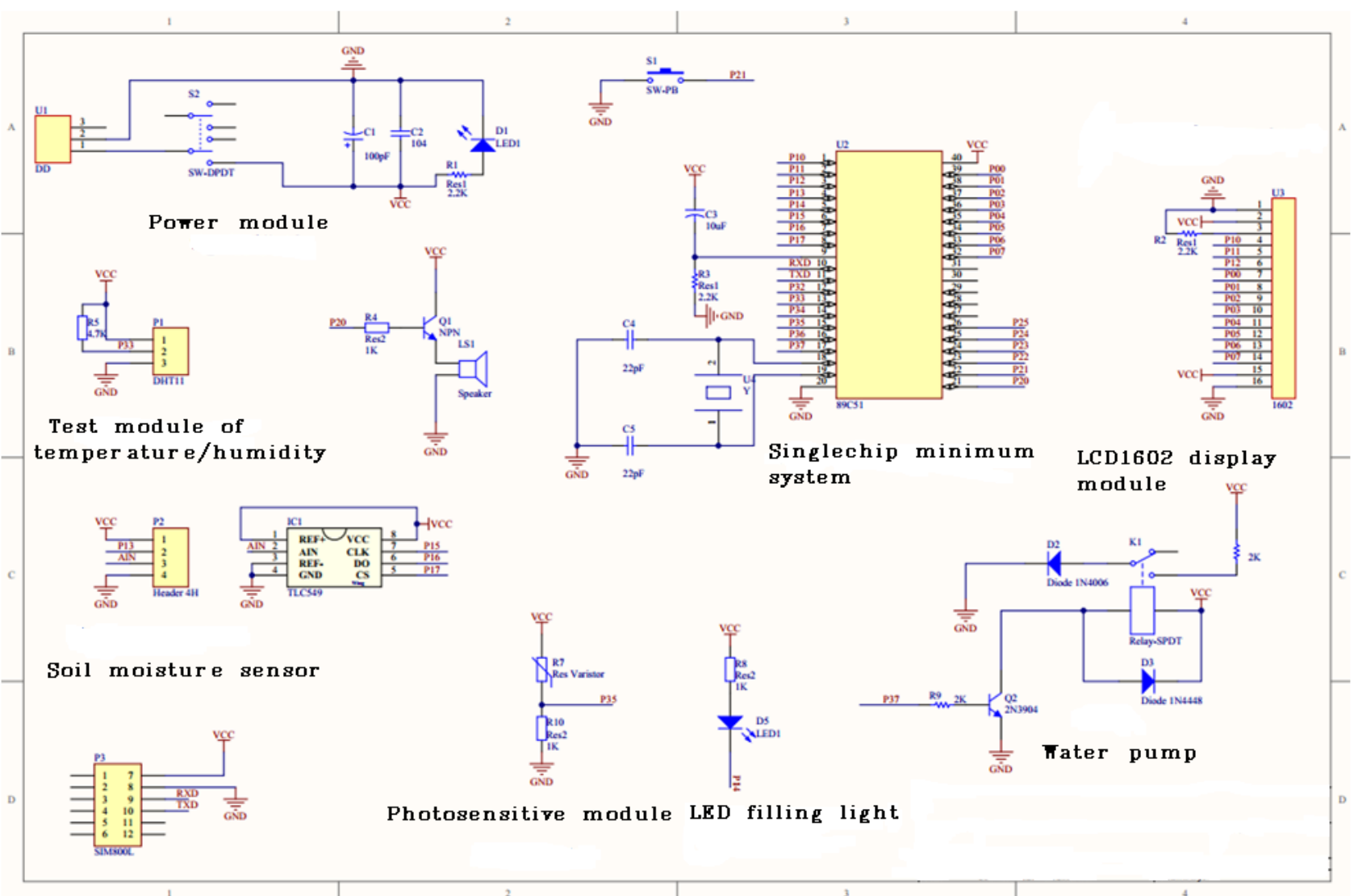

Figure 3 Control circuits of every key parts

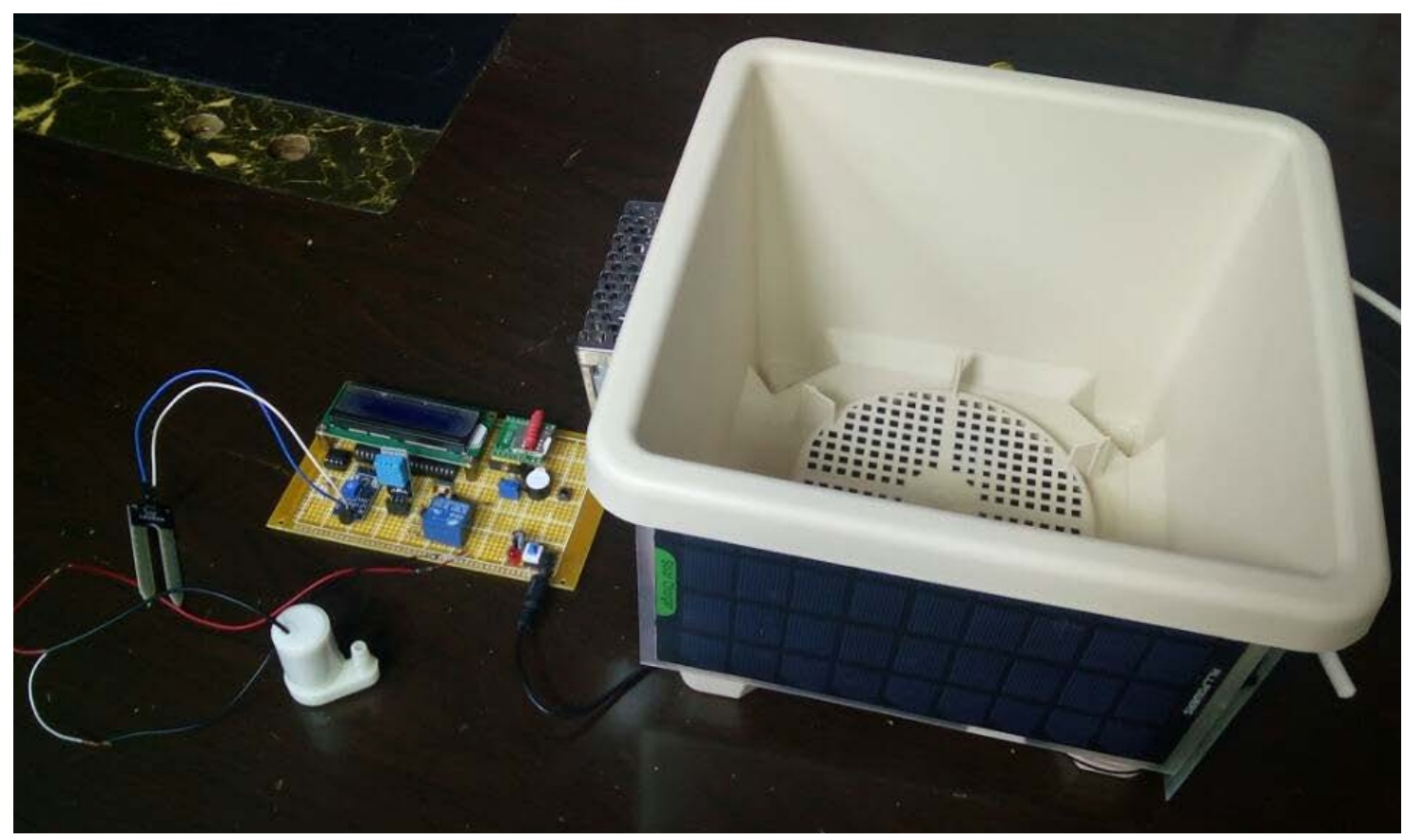

Figure 4 Prototype of the remote controlled intelligent flowerpot

\section{Conclusions}

A remote controlled intelligent flowerpot was designed in this paper for monitoring the parameters of plant growth when no person caring of flowerpot long time. The flowerpot system is made up of GSM-SM modem, LCD screen, voltage stabilizer, humidity and temperature sensor, micro-controller, water pump and so on. Tests proves that the flowerpot system is useful and it can be also used in the fields of growth of field crop, greenhouse cultivation. 


\section{References}

[1] Li H. (2016) Research and Design of Remote Controlled Intelligent Home System(in Chinese). Southwest Jiaotong University, 1-5.

[2] Li M. and Xu K.Q. (2014) Cloud Intelligent Remote Controllable Integrated Cooker Design of Smart Appliances. Design, 2, 45-46.

[3] Zhang N. (2016) The Research on Smart Flowerpot Technology. Jilin Agricultural University, $1-5$.

[4] Zhang N., Wu W.F., Du J.S., etc. (2016) Research Status and Development Prospect of Smart Flowerpot. Agriculture and Technology, 1, 174-176.

[5] Zhang J.P. and Sheng X.F. (2016) Design of Intelligent Flowerpot System Based on SCM. Electronics World, 12, 38-38.

[6] Zhang L.N., Ning H. and Liu L. (2017) Design of Intelligent Flowerpot System Based on Cloud Service. Internet of Things Technologies, 5, 76-79. 